\title{
SYNTHESIZING RESEARCH IN MARKETING THROUGH META-ANALYSIS
}

Chiara Orsingher *

The marketing field has gathered a relevant body of knowledge in the past fifty years across its various domains and sub-domains. This conspicuous body of knowledge is made up of studies that partly replicate previous findings, and partly include some novelty, in the form of different constructs, settings, and respondents. As a result, it is sometimes difficult for marketing scholars to gain a synthetic view of the relationships that hold on a given marketing phenomenon.

Consider as an example the relationship between loyalty and its antecedents: a search on Google Scholar with "antecedents of loyalty" as a keyword yields about 845 results. How can marketing scholars benefit from the knowledge generated by these different studies without incurring the risk of getting lost in this vast number of findings? A systematic way to synthesize existing research, establish the state of the art on a given domain, and foster theory building is meta-analysis. Meta-analysis is the statistical analysis of a large collection of results from individual studies for the purpose of integrating the findings (Glass, 1976). Meta-analysis in marketing studies is a powerful tool to achieve conceptual clarity and integrate various perspectives into a coherent theoretical framework that provides a rigorous explanation of marketing phenomena (Orsingher, Hogreve and Ordanini, 2016).

Although the explanation of the meta-analytic methodology is beyond the scopes of this editorial, let me briefly describe the main steps that are recommended when conducting meta-analysis.

* Associate Professor, Department of Economics, University of Bologna, email: chiara.orsingher@unibo.it.

Mercati e Competitività n. 2, 2016 


\section{Orsingher}

The first step of every meta-analysis is the definition of the theoretical domain of interest. The researcher has to specify the theoretical construct that makes the object of the analysis, what antecedents and/or consequences relate to the construct, and how the variables fits into a theoretical framework. For example, when the aim of the researcher is to study the antecedents of loyalty, customer loyalty is the focal construct, the relations between the set of antecedents are the theoretical relationships, and Relationship Marketing builds the theoretical basis (see e.g. Watson et al., 2013).

The second step involves collecting the population of studies that contain the information on the relationships of interest. This process involves retrieving published and unpublished empirical studies. For example, Watson et al. (2013) include in their meta-analysis 116 studies published in marketing journals since 1980.

The third step involves the coding of the characteristics of the retrieved studies, and the calculation of the effect size, i.e. the quantitative measure of the strength of the relationship. For each study, the researcher codes the information about the study design (e.g. experiment vs. survey), and the basic study characteristics (e.g. type of participants). Information on the type of the effect size must also be provided. Watson et al. (2013) chose correlations coefficients as the metric for their meta-analysis and converted all measures (i.e. regression coefficients, $t$ or 1 d.f. $F$ statistic) into correlations.

The fourth step requires examining the distribution of effect sizes and analyzing the impact of moderating variables. In their meta-analysis, Watson et al. (2013) describe the correlations in terms of strength, direction, statistical significance, and sample size. Additionally, they analyze the predictors of customer loyalty and identify statistically significant moderators of the relationships between customer loyalty and its antecedents.

Conducting meta-analysis might be perceived as a time-consuming, cataloguer job. Actually, performing a meta-analysis might be beneficial for several reasons. First, meta-analysis provides an inclusive and informed picture of the population of studies on a theoretical construct. Second, meta-analysis quantitatively assesses the relationships between a theoretical construct, its antecedents and/or its consequences, correcting for the distorting effects of sampling errors, measurement errors, and other artifacts that can produce the illusion of conflicting findings (Hunter and Schmidt, 2004). Thus, meta-analysis provides an unbiased estimate of the "true" effect size for each relationship under investigation. Third, metaanalysis allows investigating the role of potential moderators in explaining the variability of the relationships between variables (Orsingher, Hogreve and Ordanini, 2016). Fourth, by integrating the findings across studies, the 
relationships identified through meta-analysis can be used in path analysis and structural equation modeling to test whether the structure of the data is coherent with the theory behind the data. In this sense, path analysis within meta-analysis can be a powerful tool for reducing the number of theories that could possibly be consistent with the data, sometimes to only one theory (Hunter and Schmidt 2004).

Finally, meta-analysis can be used for theory development. Theory development involves the revision of existing theory in the effort to avoid incremental testing and to favor the development of new ideas (MacInnis, 2011). Meta-analysis allows for the revision of existing theory because it reveals the strength, the direction, and the variation of relationships between variables, thereby providing researchers' with an indication of a theory's predictive potential and practical usefulness (Aguinis et al., 2011). Meta-analysis also favors the development of new ideas. By providing a picture of the relationships among variables, and on how these variables are in relation with one another across multiple studies, researchers are offered the opportunity to generate theoretical issues that need to be explored, identify innovative problems, and to speculate on relationships and constructs that serve as the foundations for new theory (MacInnis, 2011; Aguinis et al., 2011). Thus, although theory development is a creative process distinct from meta-analysis (Hunter and Schmidt, 2004), meta-analysis provides the empirical building blocks for theory development.

The marketing field is now advanced enough to conduct meta-analyses on phenomena that rely on well-established theories but whose findings are still sparse. Therefore, applying meta-analysis would allow gaining a deeper understanding of many established constructs, their determinants and outcomes. Customer co-creation, customer search behavior, online brand communities, and relationship quality in B2B settings, are among the marketing phenomena that might benefit from a quantitative synthesis of their findings, and a reflection about their underlying theories.

To conclude, meta-analysis might help experienced researchers to better appreciate the state of the art of a given phenomenon, and to identify how they can contribute further to the field. Meta-analysis might also help $\mathrm{Ph} . \mathrm{D}$. in their early stages, because it allows them to acquire a thorough knowledge about the current state of the literature of their research topic, about the substantive and methodological orientations of past research, and about the areas that require further exploration. Lastly, since meta-analysis requires various interdisciplinary competences, it should be considered as a good opportunity to collaborate with other colleagues, to learn form each other and generate new hypotheses for further studies. 
C. Orsingher

\section{References}

Aguinis H., Dalton D.R., Bosco F.A., Pierce C.A. Dalton C.M. (2011). Meta-analytic choices and judgment calls: Implications for theory building and testing, obtained effect sizes, and scholarly impact. Journal of Management, 37, 1: 5-38.

Glass G.V. (1976). Primary, secondary, and meta-analysis of research. Educational researcher, 3-8.

Hunter J.E., Schmidt F.L. (2004). Methods of meta-analysis: Correcting error and bias in research findings. Sage.

MacInnis D.J. (2011). A framework for conceptual contributions in marketing. Journal of Marketing, 75, 4: 136-154.

Orsingher C., Hogreve J., Andrea O. (2016). Building on the past: advancing theory in services through meta-analysis. Journal of Service Management, 27, 1: 37-42.

Watson G.H., Beck J.T., Henderson C.M., Palmatier R. (2013), How conceptual differences shape the effectiveness of customer loyalty. Report Summary, Marketing Science Institute. 